\title{
BroAdBAND Power line CoMmunication: THE ChanNel AND Noise ANAlysis For A Power LINE NETWORK
}

\author{
Mukesh Kumar Varma, Zainual Abdin Jaffery and Ibraheem
}

FET, Department of Electrical Engineering, Jamia Millia Islamia, NewDelhi, India

\begin{abstract}
The scope for broadband powerline Communication is considered as a retrofit technology for wide geographical coverage wherever the human habitation exists. So during the last decade, it has drawn an enormous quantity of research work for improving communication performance and this system being standardized all over the world. The broadband power line Communication channel modelling is essential in the design of a reliable communications system. An analysis on the proposed channel model is conducted in this paper; also the paper studied the noises in Broadband powerline Communication network and its mathematical model. The channel Transfer function and Error Performance of Proposed powerline communication System noise is evaluated with various digital modulation techniques Bit error rate (BER) and signal to noise ratio (SNR) curve by using simulation software. The results indicated that the noise analysis is effective for modelling the power line communication channel. Also, we have presented the various studies on the channel performance based on Orthogonal frequency-division Multiplexing (OFDM) systems for an efficient design of a Broadband Powerline Communication (BPLC) system.
\end{abstract}

\section{KEYWORDS}

Channel model, transfer function, noise modelling, Bit error ratio, orthogonal frequency-division multiplexing.

\section{INTRODUCTION}

This time, broadband communication becomes the part and partial of our day to day life. Due to uneven geographical condition and demographic setup in the country, In remote distance hilly areas, desert areas broadband communication accesses are still a big issue. Where broadband communication lines are available development is faster and wherever it is not available, slower development in these area. The people of these areas feel they are isolated, lagging from the rest of the world. Broadband communication significantly impacts the social and economic growth of the country [1]. Alternatively, electrical power lines are available in most of these areas, so the deployment of a power line for broadband communication is economically feasible technology, as it stops additional expenditures on infrastructure for system channel. The communication technology through the power lines is now reflected as a worthy substitute for the realizing communication network. Broadband powerline communication permits easy fixing with relatively-low access costs because it needs no new wiring variations to allow broadband connection (it can be retrieved from at all outlet) and makes use of present power networks without adding a single new cable. Just plug the device into a socket-outlet and one is instantly linked to the Internet. However, the application of communication over the power transmission line is a quite old practice. From several years' power utility companies have been applying these networks for data communication purposes, measurements, control, monitoring and regulation of power plant and distribution network operation. Therefore, the low voltage power- line communication turns into a very interesting field of research. 
The Power line communication technologies can be scattered into two main kinds, Broadband, and Narrowband Communication. Broadband communication over the Powerline uses a usual for high frequency and high-speed, that can have over $100 \mathrm{Mbps}$ speed at the physical layer. Similarly, the Broadband powerline communications technology fall into two main categories; Access and In-house powerline network [1, 5-9]. A channel representation in several $\mathrm{kHz}$ to 20 $\mathrm{MHz}$ the frequency range was presented in [5]. Access power line technologies are used for sending data over the Low voltage system. Similarly, In-house powerline communication technology transfers data exclusively within the building and extends to all socket outlets within the house [10-12]. Figure (1) shows the structure of the broadband powerline communication network. In fact, the broadband powerline communication channel suffers from attention which is frequency dependent, and it is produced by line conductor and dielectric losses. The characteristics of internal in-house powerline channel transfer function are exciting to model due to the considerable volatility and complication of the networks. Vary significant efforts has been dedicated in the past to define the standard channel model for the broadband communication over powerline as a two wire transmission line [2,9]. Since the indoor (home) powerline network is quite mature than the outdoor broadband access, so we focus here on the channel modelling for low voltage in the home network, between the socket outlets.

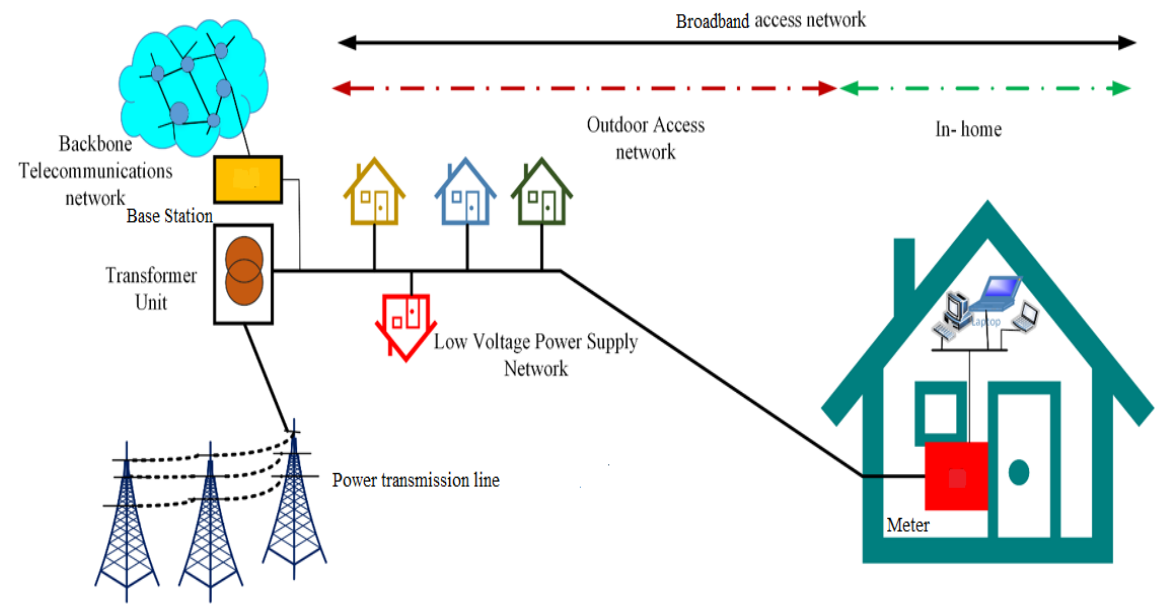

Figure 1. Structure of Broadband Powerline communication network [16]

The electrical power line was designed for transmission of power at $50 \mathrm{~Hz}(60 \mathrm{~Hz})$, having low frequency, while the broadband signal has a high frequency. The broadband Powerline Communication system has its own limits, such as it deals a challenging environment for communication. The noise, line impedance, and attenuation vary with time, frequency, and location; thus, making it challenging to model the Broadband powerline Communication channels [2].

Noise is a significant parameter to define the nature of the powerline channel; hence it is required to study the channel noise characteristics of a broadband power line system channel. The power distribution systems are an exceptional structure for a broadband data-communication, though several noises are present due to stochastic transformation in the system load impedance. Noise in the broadband powerline communication network is categorized into three leading classes, the first one is Colored background noise, second one is the narrowband noise, and the next one is impulsive noise. Background noise and Impulsive noise are the extensive kinds of additive noise in the broadband powerline Communication systems. Impulsive noise Characteristics and its cyclic dependency on the phase synchronous to main frequency are presented in [1]. Authors in [8], proposed the Narrowband noise appears from the current form of amplitude modulation of 
radio communication stations, from short, medium wave ranges. The Impulsive noises produced substantial between the existing noise categories in the broadband powerline communication networks. Some of the noise model scheme created in the available literature, whose real value normally varies vary limited, because mostly they describe the bottom-up approach, that is defining the performance of the network $[1,3,8]$. This work is an effort to discover the diverse type of noise and their analysis in a Broadband powerline communication network and also enhance the performance of the powerline channel of the system. The block diagram of the channel is offered in figure (2). The detailed Noise characteristics found in broadband powerline communication are described in section (3).

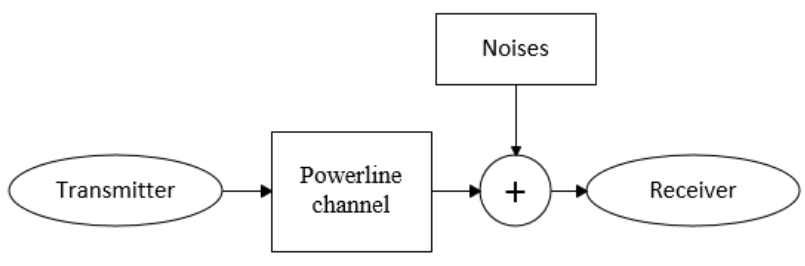

Figure 2. Block diagram of Powerline communication channel [11]

The contribution of this paper has been divided into four sections. Powerline channel model, characterization is offered in Section-2, The network channel model and Transfer function evaluation work out in this Section. Section-3 illustrated the noise analysis and error Performance of Proposed Powerline communication System. section 4 presented the OFDM modulation systems. Finally, Section-5 discusses the result and analysis as the conclusion of this paper.

\section{Power line Channel Model}

The structure of the powerline grid does not have independent one to one links between transmitter and receivers, whereas it seems like a line bus. It consists of numerous branches, joints in the house wiring cables and mismatched impedance that causes multiple reflections. In this type of case, multipath signal propagation effect must be taken into account. The simplified network topology proposed in this paper for broadband powerline communication network as it is shown in figure (3). When the power transmission line is utilized for high-frequency data communication, the signal data doesn't route the direct track between the transmitter and receiver, these consequences in Multipath signal propagation. Mingling the multipath signal propagation, frequency and line length the channel Transfer function are stated as in channel analysis [5,11]. Multipath signal propagation is considered for simple illustration which can be simply evaluated as exemplified in Figure (3). The connection has four nodes A, B, C and having only one branch and with the lengths $\mathrm{L}_{1}, \mathrm{~L}_{2}$ and $\mathrm{L}_{3}$. For the broadband powerline channel model introduced [5]. The signal transmission routes for such type grid can be inscribed as: (i.e., $\mathrm{A} \rightarrow \mathrm{B} \rightarrow \mathrm{C}, \mathrm{A} \rightarrow \mathrm{B} \rightarrow \mathrm{D}-$ $>\mathrm{B}->\mathrm{C}, \mathrm{A} \rightarrow \mathrm{B} \rightarrow \mathrm{D}->\mathrm{B}->\mathrm{D}->\mathrm{B} \rightarrow \mathrm{C}$, and so on) [17-20]. 


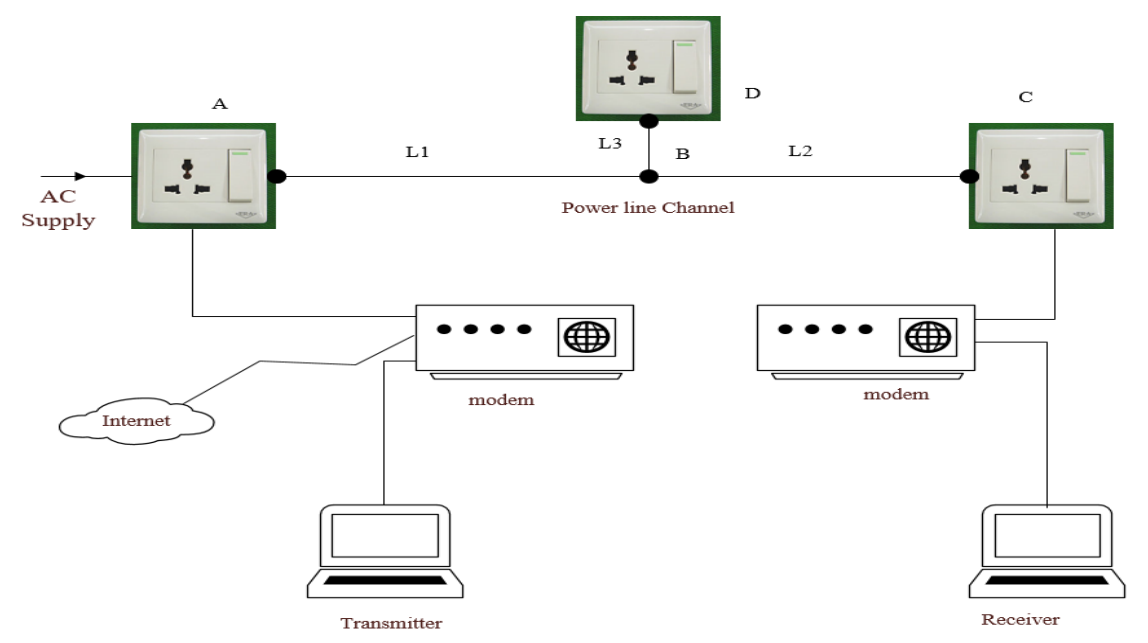

Figure 3. Model of Multipath Broadband powerline channel[12]

\subsection{Algorithms for analysis}

The factors define a Transmission lines are the characteristic impedance $\left(\mathrm{Z}_{0}\right)$ and the Propagation constant $(\gamma)[3,4]$.

The Characteristic Impedance

$$
Z_{0}=\sqrt{\frac{R+j \omega l}{G+j \omega C}}
$$

and Propagation Constant

$$
\gamma=\sqrt{(\mathrm{R}+\mathrm{j} \omega \mathrm{l}) \times(\mathrm{G}+\mathrm{j} \omega \mathrm{C})}=\alpha+\mathrm{j} \beta
$$

The real part of $\alpha$ is known as attunation factor increases with frequency, can be approximated as

$$
\alpha(f)=a_{0}+a_{1} f^{k}
$$

Where ${ }^{a_{0}}$ and ${ }^{a_{1}}$ are attunation parameters, and $\beta$ is phase constant.

The channel transfer function is specified by $[5,12]$

$$
\mathrm{H}(\mathrm{f})=\sum_{i=1}^{N} \mathrm{~g}_{\mathrm{i}} \times\left(e^{\left[-\left(a_{0}+a_{1} f^{K}\right) d_{i}\right]} \times e^{\left[-2 \pi \mathrm{f}\left(\frac{d_{i}}{v_{p}}\right)\right]}\right)
$$

Where $\mathrm{N}$ is the number of multi paths, the $\mathrm{g}_{\mathrm{i}}$ is Weighting factor, $\mathrm{d}_{\mathrm{i}}$ is path length of $\mathrm{I}^{\text {th }}{ }^{\text {th }}$ path, $\mathrm{a}_{0}$ and $\mathrm{a}_{1}$ is attunation parameters, and $\mathrm{v}_{\mathrm{p}}$ phase velocity, f-frequency and $\mathrm{k}$ - exponent of attunation factor (0.2-1.0). Part of the equation $e^{\left[-\left(a_{0}+a_{1} f^{K}\right) d_{i}\right]}$ is an attenuation portion and the part $e^{\left[-2 \pi f\left(\frac{d_{i}}{v_{p}}\right)\right]}$

is known as delay portion. The wiring follows different-different norms in many places around the country, so measurements from all location would be necessary. 


\subsection{Preparation of Data and its Investigative analysis}

In this analysis, the variety of cable used is common in India for house wiring; the cross section of cable is illustrated in figure (4). The powerline cables are made up of two copper conductors with inside PVC wall, which can be similar to as a proximate form of the "two-wire transmission line".

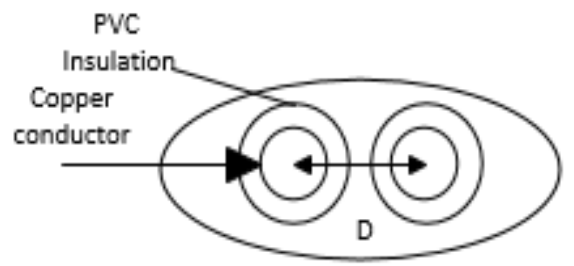

Figure 4. The structure of a typical single-phase low voltage power cable [11]

Table 1. shows the characteristics parameters estimated for different cables of network.

\begin{tabular}{|c|c|c|c|c|c|}
\hline Types of cable & $\mathbf{0}$ & $\mathbf{1}$ & $\mathbf{2}$ & $\mathbf{3}$ & $\mathbf{4}$ \\
\hline Cross section in $\left(\mathrm{mm}^{2}\right)$ & 1.50 & 2.50 & 4.0 & 6.0 & 10 \\
\hline$\varepsilon_{\text {eq }}$ & 1.45 & 1.52 & 1.56 & 1.73 & 2.00 \\
\hline Char. Impedance $\mathrm{Z}_{0}$ in $(\Omega)$ & 270 & 234 & 209 & 178 & 143 \\
\hline Resistance $\mathrm{R}_{0}$ in $(\Omega)$ & 12.0 & 9.34 & 7.55 & 6.25 & 4.98 \\
\hline Inductance $\mathrm{L}$ in $(\mu \mathrm{H} / \mathrm{m})$ & 1.08 & 0.96 & 0.87 & 0.78 & 0.68 \\
\hline Capacitance in $(\mathrm{pF} / \mathrm{m})$ & 15.0 & 17.5 & 20.0 & 25.0 & 33.0 \\
\hline Conductance $\mathrm{G}_{0}$ in $(\mathrm{s} / \mathrm{m})$ & 30.9 & 34.7 & 38.4 & 42.5 & 49.3 \\
\hline
\end{tabular}

Table 2. The attunation parameters corresponding to the length profiles [2].

\begin{tabular}{|c|c|c|c|c|}
\hline Class & $\mathbf{g}_{\mathbf{i}}$ & $\mathbf{a}_{\mathbf{0}}\left(\mathbf{m}^{-\mathbf{1}}\right)$ & $\mathbf{\mathbf { a } _ { 1 }} \mathbf{( \mathbf { s } / \mathbf { m } )}$ & $\mathbf{k}$ \\
\hline $100 \mathrm{~m}$ & 1.0 & $(9.40) 10^{-3}$ & $(4.20) 10^{-7}$ & 0.70 \\
\hline $150 \mathrm{~m}$ & 1.0 & $(1.09) 10^{-3}$ & $(3.36) 10^{-7}$ & 0.70 \\
\hline $200 \mathrm{~m}$ & 1.0 & $(9.33) 10^{-3}$ & $(3.24) 10^{-7}$ & 0.70 \\
\hline $300 \mathrm{~m}$ & 1.0 & $(8.40) 10^{-3}$ & $(3.00) 10^{-9}$ & 1.00 \\
\hline $380 \mathrm{~m}$ & 1.0 & $(6.20) 10^{-3}$ & $(4.00) 10^{-9}$ & 1.00 \\
\hline
\end{tabular}

Where $\mathrm{R}=\mathrm{R}_{0} \cdot 10^{-5} \cdot \sqrt{\mathrm{f}}(\Omega$ per metre $)$ and $\mathrm{G}=\mathrm{G}_{0} \cdot 1 \cdot 10^{-14} \cdot 2 . \pi . \mathrm{f}(\mathrm{s} / \mathrm{m})$

\subsection{Channel Transfer Function Response}

In the paper, a dynamic model of powerline is considered for investigations. The cable data used in this case is a two conductor Copper conductor of diameter $8 \mathrm{~mm}$, the space between the conductor is 0.15 meter, PVC insulated. It is the cable which is commonly available for housewiring. Based on the transfer function equation (4), A software tool for obtaining the channel transfer function is developed. Also, the structure for the typical indoor broadband powerline communication network channels are shown in figure 2 is investigated; its performance is illustrated in Fig. 4. Lengths of sections $\mathrm{L}_{1}, \mathrm{~L}_{2}$ and $\mathrm{L}_{3}$ are $30 \mathrm{~m}, 170 \mathrm{~m}$ and $11.5 \mathrm{~m}$ respectively. The rest parameters are adopted from [2] as in table 3 and 4. Simulation is showed at the example that the number of paths $\mathrm{N}$ is 4 and 15 . In the part of equation (4), the attenuation term of the channel model, the constraint sets at $\mathrm{k}=1, \mathrm{a}_{0}=0$ and $\mathrm{a}_{1} 7.8 \times 10-10 \mathrm{~s} / \mathrm{m}$. [2]. Figure (5) illustrated the channel frequency response plot for 4,15-paths, indicates that the varying trends of the transfer function in the frequency series of 1-30 MHz. 
Table 3. path parameters (for $\mathrm{N}=4$ ) [2]

\begin{tabular}{|l|l|l|l|l|}
\hline $\mathbf{N}$ & $\mathbf{1}$ & $\mathbf{2}$ & $\mathbf{3}$ & $\mathbf{4}$ \\
\hline $\mathrm{g}_{\mathrm{i}}$ & 0.64 & 0.38 & -0.15 & 0.05 \\
\hline $\mathrm{d}_{\mathrm{i}}$ & 200 & 223 & 246 & 269 \\
\hline $\mathrm{N}$ & 1 & 2 & 3 & 4 \\
\hline $\mathrm{g}_{i}$ & 0.64 & 0.38 & -0.15 & 0.05 \\
\hline $\mathrm{d}_{i}$ & 200 & 223 & 246 & 269 \\
\hline
\end{tabular}

Table 4. path parameters (for $\mathrm{N}=15$ ) [2]

\begin{tabular}{|l|l|l|l|l|l|l|l|l|}
\hline $\mathbf{N}$ & $\mathbf{1}$ & $\mathbf{2}$ & $\mathbf{3}$ & $\mathbf{4}$ & $\mathbf{5}$ & $\mathbf{6}$ & $\mathbf{7}$ & $\mathbf{8}$ \\
\hline $\mathrm{g}_{\mathrm{i}}$ & 0.029 & 0.043 & 0.103 & -0.058 & -0.045 & -0.040 & 0.038 & -0.038 \\
\hline $\mathrm{d}_{\mathrm{i}}$ & 90 & 102 & 113 & 143 & 148 & 200 & 260 & 322 \\
\hline $\mathbf{N}$ & $\mathbf{9}$ & $\mathbf{1 0}$ & $\mathbf{1 1}$ & $\mathbf{1 2}$ & $\mathbf{1 3}$ & $\mathbf{1 4}$ & $\mathbf{1 5}$ & \\
\hline $\mathrm{g}_{\mathrm{i}}$ & 0.071 & -0.035 & 0.065 & -0.055 & 0.042 & -0.059 & 0.049 & \\
\hline $\mathrm{d}_{\mathrm{i}}$ & 411 & 490 & 567 & 740 & 960 & 1130 & 1250 & \\
\hline
\end{tabular}

In the Broadband powerline communication environment, signal distortion is expressed not only by channel transfer function $\mathrm{H}(\mathrm{f})$, but noise play an important role in it. As the noise signal is produced by interferences and inhabits part of the frequency band. The short wave communications generate noise and may affect the powerline communications.

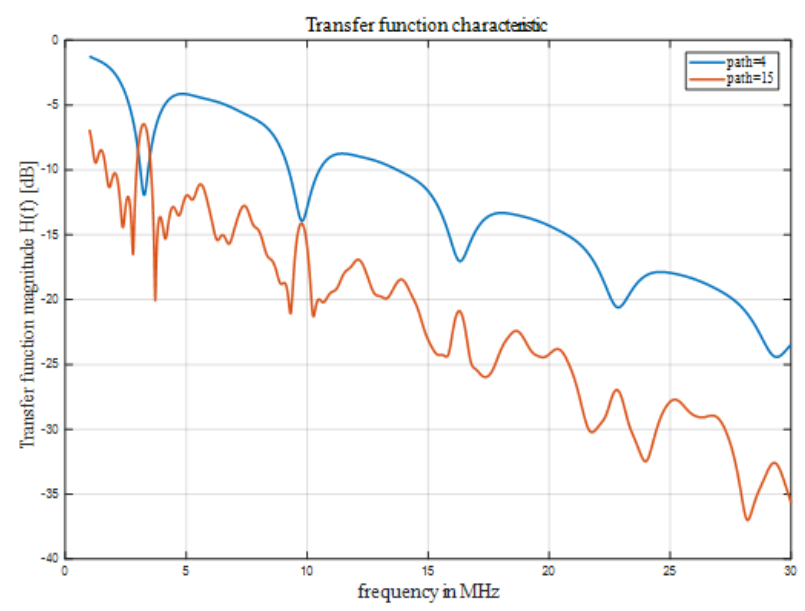

Figure 5. Simulated Transfer functions characteristics for $\mathrm{N}=4,15$-path

\section{Power line Channel Noise Analysis}

Noise in a communication system can be explained as any undesired signal which can prove an obstruction to the needed signal. In this paper, we mainly focus on the noise generated by voltage level, by the electrical devices, switching operation and network faults. The Power line networking is likewise susceptible to nosiness from devices linked to that power supply infrastructure, for example, Fluorescent tube lights, washing machine, drill, hair dryers, microwave ovens, computers, switch mode power supply etc. may generate, noise on the power line network. So there is a mass of noise sources; it can often be caused by external disturbances 
and can lead to errors in a communication system [1-4]. Broadband transmission over a powerline channel is mainly affected by the ever-present noise. It means similar to the other communication channels, broadband Powerline Communication atmosphere is highly exaggerated by noise, attenuation, and line impedance of channel. In Broadband powerline communication networks the noise signal has to be measured in different positions of the network. Generally, in low/ medium voltage access Networks, measurements are made at transformation centres or substations, and in Home powerline communication it measured at the central meters or at the consumer socket outlet point. This is reflected in the most important positions of the network.

The noise features are significant constraints to define the nature of powerline communication channel interference. This noise can be considered into numerous classes reliant on its source, level amount and the time domain signature [10], as the coloured Background noise, Narrowband noise, Periodic impulsive noise (Synchronous or Asynchronous to the frequency of main supply), besides the aperiodic impulsive noise. The declining of all the noise coming from electrical powerline network is critical. Powerline noise is indicated in the block diagram of Figure (6).

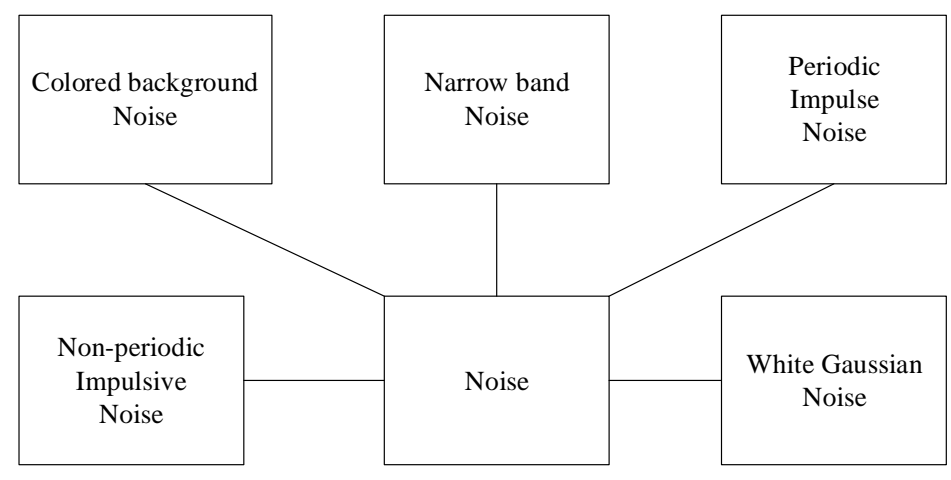

Figure 6. Types of Noise

The colored background noise

$$
\left[n_{c G}(\mathrm{f})\right]=N_{\infty}+N_{0} e^{\frac{f}{f_{0}}}
$$

Where $\left[n_{c G}(\mathrm{f})\right]$ is the power spectral density of colored background noise, $N_{\infty}$ is constant noise density $N_{0}$ and $\mathrm{f}_{0}$ are the constraints of the exponential function.

And the narrowband noise

$$
\left[n_{n b}(f)\right]=\sum_{i=1}^{N} A_{i}(t) \cdot \sin \left(2 \pi f_{i} t+\varphi\right)
$$

where $\mathrm{N}$ indicates the entire number of narrowband interferes, and $\mathrm{A}_{\mathrm{i}}(\mathrm{t}), \mathrm{f}_{\mathrm{i}}$, and ${ }^{\varphi}$ designate the amplitude, frequency level and the phase of the conventional narrowband noise correspondingly. The model of the colored noise is similar to the Additive White Gaussian noise (AWGN). The power spectral density of background noise $\left[n_{b g}\left(f_{)}\right)\right.$is the sum of colored background noise [ $n_{c G}$ (f) ] and narrowband noise [ $n_{n b}$ (f) ] can be expressed from (5) and (6) as

$$
\left[n_{b g}\left(f_{)}\right)=\left[n_{c G}(\mathrm{f})\right]+\left[{ }_{n b}(\mathrm{f})\right]\right.
$$

Signal to noise ratio is a measure to quantify how much a signal has been corrupted by noise and calculated as

$$
\text { Signal to Noise ratio (SNR)in } \mathrm{dB}=10 \log _{10}\left(\frac{\mathrm{P}_{\text {signal }}}{\mathrm{P}_{\text {Noise }}}\right)
$$

The signal to noise ratio higher than $0 \mathrm{db}$, designates higher signal power than the noise power. 


\subsection{Error Performance of Proposed Communication System}

This structural design has been extensively researched with broadband power line communications by scientists and engineers in the research world. The Broadband powerline communication system is a complicated one and using model for functional blocks such as different kinds of modulator-demodulator, encoder and decoder, and all kinds of signal source, channel model, noises, filters, amplifiers etc. The channel model shown in figure (7) shows only a subset of the functional blocks in broadband powerline communication.

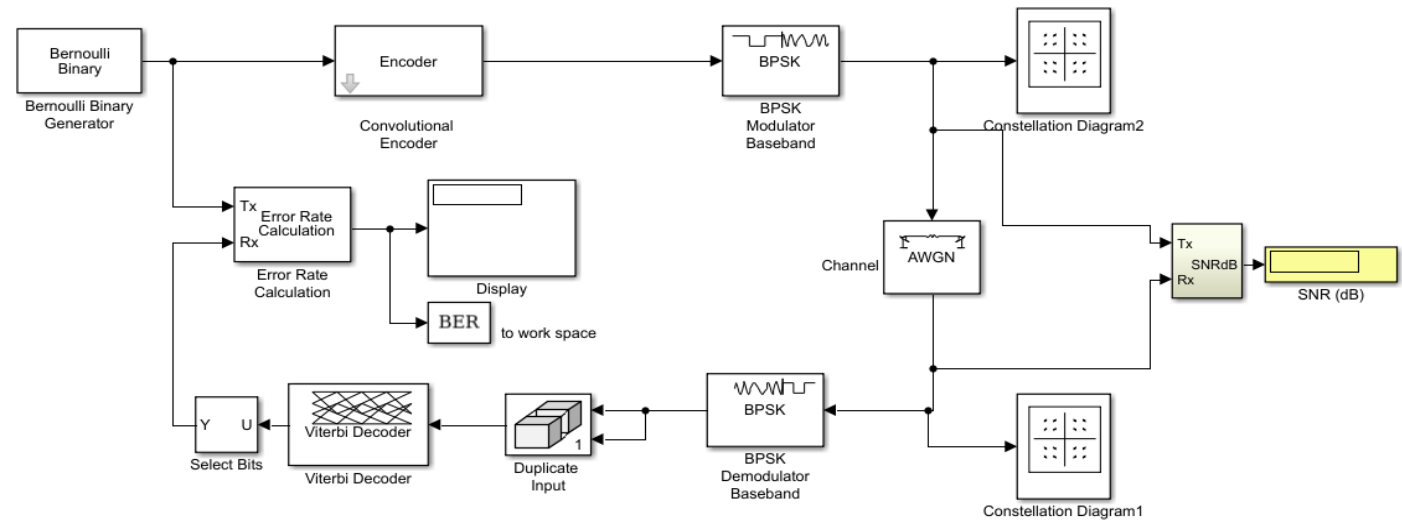

Figure 7. Functional blocks in broadband powerline communication

In this segment, we present numerical simulation results that show the system error performance of the proposed powerline communication networks. The theoretical and calculated signal to noise ratio (SNR) and bit error rate (BER) of the proposed powerline channel under BPSK and QPSK, the curve is drawn using 'bertool' in MATLAB for AWGN environment. In both the scheme, an improvement in BER has been observed. QPSK scheme is always preferred to BPSK in multipath fading channels. Figure (8) below presents BER and SNR performance curve in the AWGN channel.
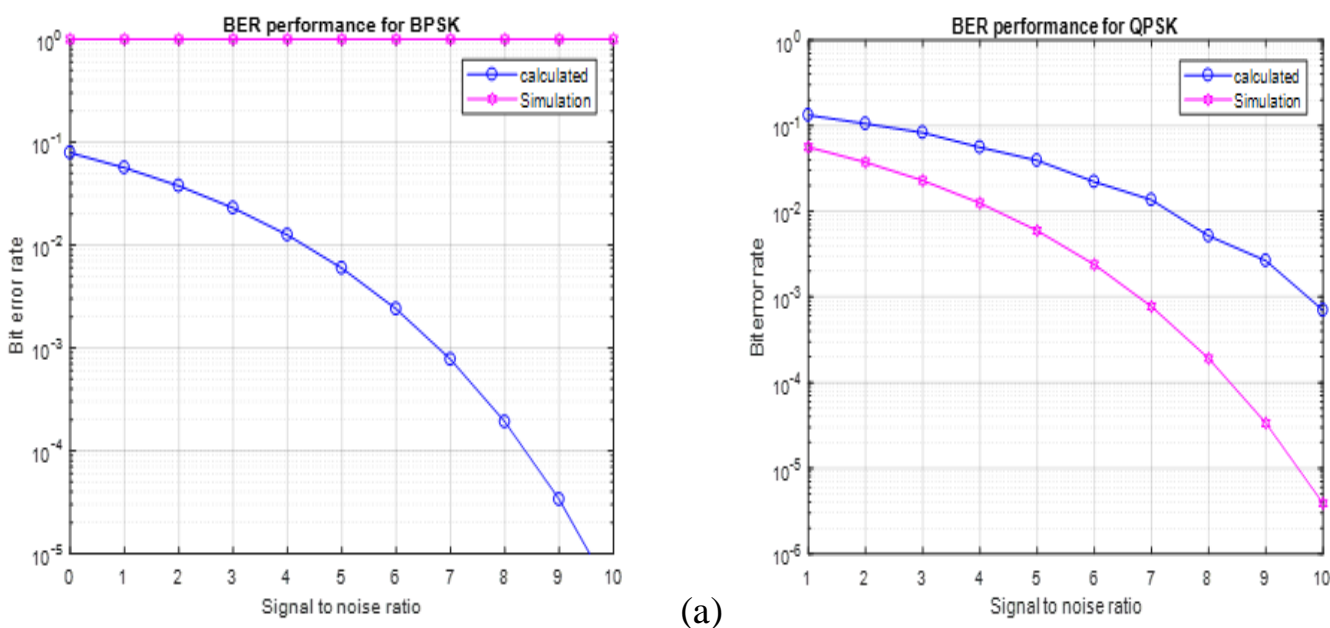

(a)

Figure 8. BER performance curve for BPSK and QPSK in AWGN channel 


\section{OFDM SYSTEM}

Orthogonal frequency division Multiplexing (OFDM), is the succeeding technology for high speed communication system. In the previous section, we analyzed Error Performance of Proposed Communication System. OFDM system model has been adopted for BPLC communications as it gives better performance at high frequencies used in BPLC [13-17]. OFDM is a special form of the frequency-division multiplexing (FDM) multicarrier modulation technique. In multicarrier systems, the information to be transmitted is split into multiple smaller chunks and transmitted independently. Each of these subcarriers contains numbers of parallel data streams or channels and is modulated conventionally at a low symbol rate; these are groups of bits of data related to (but not the same as) gross bitrate, which is expressed in bits/second. The single carrier, multi-carrier, and OFDM frequency spectral curve is shown in figure (9) below [14].

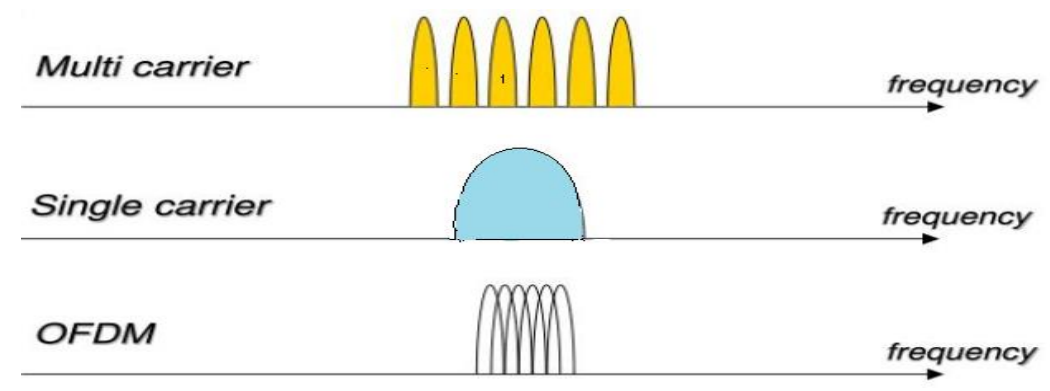

Figure 9. OFDM spectrum curve[14]

The OFDM modulation techniques are found to provide better error handling, efficient use of bandwidth and reduction in inter-symbol and inter-channel interference [13]. Now here we have discussed the various studies on the channel performance based on orthogonal frequency-division Multiplexing (OFDM) systems as in BPLC system, OFDM modulation is used. The use of orthogonal frequency division Multiplexing (OFDM) is also considered a critical feature allowing broadband powerline communication (BPLC) to avoid certain frequencies. Orthogonal frequency division Multiplexing (OFDM) uses numerous orthogonal subcarriers to transmit the data, it also provides improved robustness beside frequency selective fading and Narrowband interference, and is efficient in distributing with multi-path delay spread [17-18]. In figure (10) shows the block diagram of broadband powerline communication with OFDM transmission.

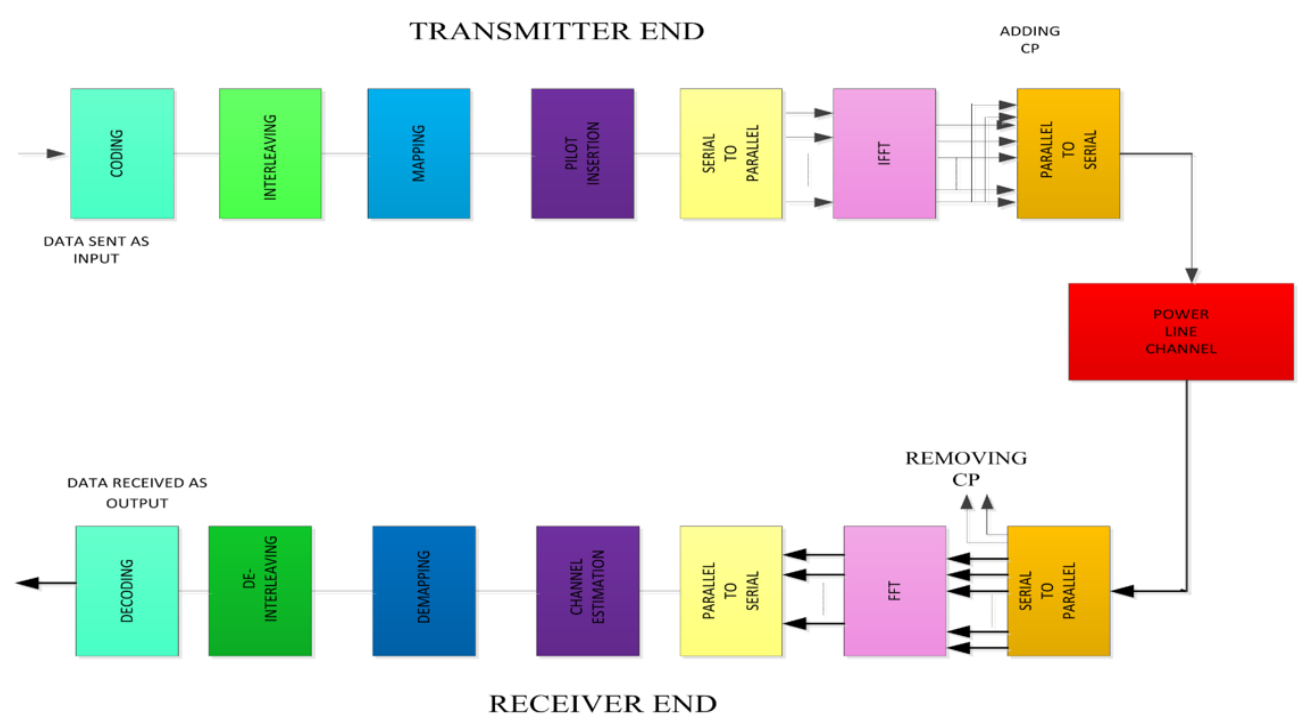

Figure 10. BPLC communication model with OFDM transmission [16] 


\section{RESUlt AND CONCLUSIONS}

The broadband powerline communication technology is an exciting alternative for High-speed data communication, the work carried out for the channel analysis for the proposed channel model. The analysis of both the channel and the noise is obtained from limited data and the transfer function and Error Performance of Proposed channel model are simulated by using MATLAB software. The simulation results show the increase of power line length is produced deep notches which signify attenuation on the broadband power line communication Channel. Further, this paper is evaluated noise error performance with various digital modulation techniques. The results indicate that the channel Transfer function and Noise analysis is effective for modelling the of power-line Communication channel. OFDM is an attractive modulation scheme for the high data rate BPLC applications.

\section{ACKNOWLEDGEMENTS}

The authors would like to acknowledge the reviewers for their valued remarks and counsels, which provided valuable direction as to resolve errors done while writing this research paper, how we could improve this paper. We are very thankful for suggestions to improve the paper. The first author is also thankful and grateful to Jamia Millia Islamia, New Delhi and G.B. Pant Institute of Technology, New Delhi for providing me an opportunity to work on this research work.

\section{REFERENCES}

[1] Zimmermann M.and Klaus Dostert (2002) "A multipath model for the power line channel" IEEE Trans. On Communications, vol.50, N0.4, pp.553-559.

[2] Manfred Zimmermann, Klaus Dostert (1999), A Multi-Path Signal Propagation Model for the Power Line Channel in the High Frequency Range, ISPLC, UK, pp. 44- 51.

[3] H. Meng, S. Chen, Y. L. Guan, C. L. Law, P. L. So, E. Gunawan, and T. T. Lie (2004), "Modeling of transfer characteristics for the broadband power line communication channel" IEEE Transactions on Power Delivery, Vol.19, N0.3, pp.1057-1064

[4] H. Meng, S. Chen, and Y. L. Guan, (2005) "Modeling and Analysis of Noise Effects on Broadband Power-Line Communications" IEEE Transactions on Power Delivery, Vol.20, N0.2, pp.630-637.

[5] Zimmermann M. and Klaus Dostert (2002) "Analysis and modeling of impulsive noise in broadband power line communications" IEEE Transactions of Electro-magnetic compatibility, vol. 44, N0.1, pp.249-258.

[6] H. Phillips, (1999)," Modeling of powerline communication channels", in Proceedings of International Symposium on Power Line Communications and Its Applications (ISPLC 99), UK, pp.14-21.

[7] Papadopoulos T.A., Christos G. Kaloudas, A.I. Chrysochos and Grigoris K. Papagiannis (2013)," Application of narrowband power line communication in medium voltage smart distribution grids", IEEE Transactions on Power Delivery, vol.28 (2), pp.981-988.

[8] Papadopoulos T.A., A.I. Chrysochos and Grigoris K. Papagiannis (2013) "Narrowband power line communication: Medium voltage cable modeling and laboratory experimental results", ELSEVIER, Electric power systems research, 102, pp.50-60.

[9] S. Galli, T. Banwell, (2006) "A deterministic frequency-domain model for the indoor power line transfer function", IEEE Journal on Selected Areas in Communications 24 (7), pp.1304-1316. 
[10] P. Amirshahi, F. Canete, K. Dostert, S. Galli, M. Katayama, M. Kavehrad, (2010) "Power Line Communications: Theory and Applications for Narrowband and Broadband Communications over Power Lines,"1st Edition, Wiley.

[11] Mukesh Kumar Varma, Zainul Abdin Jaffery and Ibraheem, (2015) "Advances of Broadband power line communication and its application”, 12'th IEEE International Conference INDICON, JMI, New Delhi, India, pp. 17-20.

[12] Mukesh Kumar Varma, Zainul Abdin Jaffery and Ibraheem, (2018) "A comprehensive study on channel modeling of broadband communication over low voltage power line" proceeding IEEE sponsored 5'th International Conference on Signal Processing and Integrated Networks, SPIN 2018, Amity University, Noida, India, pp.22-23.

[13] Justinian Anatory, Nelson Theethayi and Rajeev Thottappillil, (2009) "Effects of Multipath on OFDM Systems for Indoor Broadband Power-Line Communication Networks", IEEE Transactions on power delivery, vol. 24, no.3, pp. 1190-1197.

[14] Andrea M. Tonello, Salvatore D’Alessandro, and Lutz Lampe, (2010) “Cyclic Prefix Design and Allocation in Bit-Loaded OFDM over Power Line Communication Channels", IEEE Transactions on Communications, vol. 58, no. 11, pp. 3265-3276.

[15] Anatory Justinian, Nelson Theethayi and Rajeev Thottappillil (2009) "Performance of Underground Cables That Use OFDM Systems for Broadband Power-Line Communications" IEEE Trans. On Power Delivery, vol.24, N0.4, pp. 1889-1897.

[16] Seema Arora, Vinay Kumar Chandna and Mini S. Thomas, (2012)" Performance Analysis of 16QAM using OFDM for Transmission of Data over Power Lines " ELSEVIOR, Energy Procedia 14, pp.1723-1729.

[17] Konark Sharma and Lalit Mohan Saini, (2015)" Performance analysis of smart metering for smart grid: An overview "ELSEVIER Journal of Renewable and Sustainable Energy Reviews 49, pp.720735 .

[18] Philipps, H.,(1999) "Modelling of powerline communication channels" international Symposium on Power-Line Communications (ISPLC '99), Lancaster, UK, pp. 14-21.

[19] Philipps, H., (2000) " Development of a Statistical Model for Powerline Communication Channels" in proceedings of IEEE International Symposium on Power-Line Communications and its application (ISPLCA), pp. 153-160.

[20] Mukesh Kumar Varma, Zainul Abdin Jaffery and Ibraheem Nasiruddin (2018) "Analysis of High Performance Low Power Broadband Powerline Channel using S-Parameter" International journal of Research in Electronics and computer Engineering, IJRECE Vol. 6, Issue 3, ISSN: 2393-9028 (Print) | ISSN: 2348-2281 (Online). 


\section{AUTHORS}

Zainual Abdin Jaffery is professor and Head of the department in Electrical Engineering Department at the Jamia Millia Islamia New Delhi. He received his B.Sc. (engg.), M.Sc.(engg) from AMU. Aligarh in 1987 and 1989 respectively, and Ph.D. degree in Digital signal processing from Jamia Millia Islamia, New Delhi in 2004. Professor Jaffery is a senior member of the IEEE society. He is reviewer for several reputed journals and conferences, he is author and co-authored of around 50 technical papers. His research interest in Digital signal processing, Image processing, and Digital system design.

Ibraheem is senior professor at Jamia Millia Islamia, New Delhi and having about 29 years' academic-teaching experience. Presently he is working as Full professor at Qassim University, Arabia. He received his B.Sc. M.Sc.(engg.), Ph.D. from AMU, Aligarh in 1982,1987 and 2000 respectively. He is author and co-author of about 203 research papers in reviewed journals and international conferences and published one book on recent trends on power system management. He is life members of ISTE, IEEE society. His research interests include power system operation and control.

Mukesh Kumar Varma received his BE (Electrical Engineering) in 1993 from RDVV, Jabalpur (M.P.) and M. Tech.in 2011 from Jamia Millia Islamia, New Delhi. He is a Ph.D. student at Department of Electrical Engineering, Faculty of Engineering and Technology, Jamia Millia Islamia, New Delhi. He is currently working as Senior Lecturer with G.B. Pant Institute of Technology, under Directorate of Training and Technical Education, New Delhi. His research interests and research activity are focuses on power line communications and powerline channel modelling.
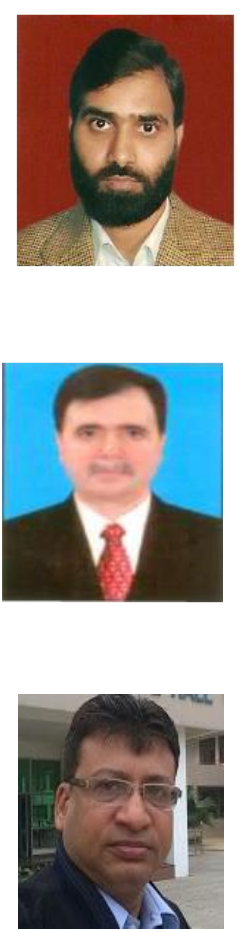